\title{
Reply to Commentary
}

\section{Nicholson, Martelli and Zasler reply to Green}

Dr. Green [1] suggests, in his letter regarding our article [2] that effort rather than other factors may account for much of the variance in many neuropsychological studies. Indeed, effort is suggested to possibly be more important than actual neurologic insult/lesion, chronic pain, sleep disorder, affective distress or other factors. Whereas we agree that an effect of effort is certainly very important to consider, we have a number of reservations about this perspective. Firstly, there appears to be poor differentiation about what is meant by effort. In many contexts this is presented as an exaggeration of difficulties often with the more or less explicit suggestion that there is conscious or wilful dissimulation, i.e., malingering. However, an effect of effort may also be associated with various other factors including actual neurologic lesion (usually involving frontal system dysfunction), sleep disturbance, chronic pain, affective distress (especially perhaps depression), various psychiatric disorders, or various other factors in which there is some interference with optimal motivational readiness or effortful processing capacity. In such cases, there may be temporary improvement in performance in response to specific prompts but the baseline level of activity remains depressed. As such, an impairment of effortful processing is part and parcel of the disorder, not merely a manifestation of wilful dissimulation.

It should be noted that virtually any competing stimulus will interfere with effortful processing. If one is suffering truly severe pain, high anxiety, somatic preoccupation, feelings of guilt or despair, etc., we think it is not unreasonable to expect that there will be an effect on neuropsychological test results due to interference with effortful processing or other aspects of processing capacity. Numerous functional neuroimaging studies of pain, depression or other psychiatric disorders show an effect on brain function. There is clearly an underlying neurobiological basis, likely modifiable and dynamic rather than static, for many such problems. Whereas some may resolve with appropriate interventions (e.g., pharmacological, cognitive-behavioral, rest, etc.), others such as those involving frontal system lesion may not. In terms of psychometric studies, several studies of acute pain with normal controls, in no way influenced by litigation status, show marked effects of pain on several measures. The same is true for the effects of depression, sleep disturbance etc. Whereas these psychometric difficulties may be partly due to an effect on effortful processing, they should not merely or necessarily be considered to be due to wilful dissimulation.

The conclusion of poor effort typically rests upon the results of certain measures deemed to assess this. The development and validation of such tasks typically involves comparison between certain groups such as healthy normal controls or certain neurologic groups attempting to do their best, groups that are instructed to attempt dissimulation, and groups or subsets of groups that may be suspected as not putting forth full effort, possibly related to wilful dissimulation, i.e., subjects involved in litigation or being assessed for the purposes of compensation. Cut-offs are typically established and when performance falls below such levels, it is often concluded that there has been poor effort or dissimulation. However, such cut-off scores virtually never take into consideration what the effects of pain, fatigue, affective distress or other such factors may have on testing. One of the few studies that have attempted to independently assess the effect of one such confounding variable (affective disorder) was that of Binder and Willis [3] who found that level of performance was significantly depressed in association with affective disorder despite there being no involvement in litigation. It should also be noted that poorer scores on the part of subjects involved in compensation may reflect actual difficulty rather than being due to dissimulation given that these subjects may be a subset with problems (e.g., post-traumatic headache, psychiatric disorder, etc.) that cause actual impairment leading to litigation/compensation. As such, we think that inferences concerning effort, especially wilful dissimulation, may often be invalid when using usual cut-offs. Such a practice may do great disservice to clients and may tarnish the reputation of professionals utilizing such methods. However, we do agree that poor performance on such 
measures of "effort" may invalidate results in terms of making inferences concerning brain-behaviour relationships in terms of common neurologic lesions.

In conclusion, we do appreciate the importance of assessing effort and poor effort associated with noncompliance (possibly related to malingering) but we think that considerable further study with (likely) novel techniques will be necessary to successfully disambiguate relevant factors in the future.

\section{References}

[1] P. Green, Comment on article "Does Pain Confound Interpretation of Neuropsychological test Results?" NeuroRehabilitation 16 (2001), 305-306.

[2] K. Nicholson, M.F. Martelli and N.D. Zasler, Does Pain Confound Interpretation of Neuropsychological test Results? NeuroRehabilitation 16 (2001), 225-230.

[3] L.M. Binder and S.C. Willis, Assessment of motivation after financially compensable minor head trauma, Psychological Assessment 3 (1991), 175-181. 